\title{
Flexible Printed Switch Integrated with Wireless Module for IoT applications
}

\author{
Jeet Kumar Brahma \\ Centre for Energy, IIT Guwahati \\ Guwahati -781039, India \\ jeet18@ iitg.ac.in \\ Charu Monga \\ Department of Design, IIT Guwahati \\ Guwahati -781039, India \\ charum@ iitg.ac.in
}

\author{
Brijesh Meena \\ Centre for Energy, IIT Guwahati \\ Guwahati-781039, India \\ meena.brijesh@iitg.ac.in \\ Harsh Chaturvedi \\ Centre for Energy, IIT Guwahati \\ Guwahati- 781039, India \\ harshc@iitg.ac.in
}

\author{
Rajat Srivastava \\ Dept. of ECE, NIT Sikkim \\ Ravangla, Sikkim \\ rajatsriva98@gmail.com
}

\begin{abstract}
This paper demonstrates a designed and fabricated flexible, printed electronic touch switch, integrated with wireless module for both manual and remote access control of the electronic appliances. Flexible switch was screen printed using silver nanoparticle and functional polymer based conductive ink. The foldable flexible switch was then integrated with the designed and assembled wireless module. Remote wireless control of the assembled integrated system demonstrates the proof of concept of integration of flexible printed capacitive touch switches with IoT based home automation modules. The integrated module described here provides smart alternative to mechanically controlled conventional switches with flexible printed switches which can be wirelessly controlled and are IoT enabled for smart home automation providing integrated automated system to remotely access and control electronic appliances. This integrated system provides proof of the concept for minimizing space and optimization of power consumption through wireless appliance control using flexible electronic switch.
\end{abstract}

Keywords- Flexible printed circuits, Wireless control circuits, IoT, Energy management.

\section{INTRODUCTION}

The rapid growth of flexible printed circuits in commercial appliances are noticeable in today's market. The aspects of this paper is to introduce the idea of microcontroller integrated flexible printed switch and its integration with IoT. The replacement mechanical switch with microcontroller based flexible printed switch provides a lot of advantages such as light weight, reduced space, no moving parts, flexibility of switch location and ability to adjust in non-uniform surfaces [1]. It also provides safety and isolation from the main power, multiple state control action with a single switch and may also acts as a low cost alternative for touch screen switches in inexpensive low cost electronic gadgets. The addition of microcontroller and concept of IoT in general switch box allows ease to explore several automated control applications like auto-timing, auto regulation, auto-sleep, wireless distant control actions, etc. [2][3]. This simple replacement could prove very beneficial in the field of automation and generate more flexibility in functionality of several local systems and appliances that does not use microcontrollers in themselves. It also allows a user to design, manage and automate the power consumption in simple appliances attached with proposed switch box to gain a better energy managed system and contribute a step towards energy saving [3].

\section{METHODS}

\section{A. Silver particles based conductive ink}

The conductive ink was developed using silver particles of the size 2-5 microns. These Silver particles were purchased from Auto Fiber Craft, Jamshedpur. Polyvinyl acetate (PVAc) polymer, Amyl Acetate and Methyl Ethyl Ketone were purchased form Sigma Aldrich. Amyl Acetate and Methyl Ethyl Ketone were mixed at 1:1 wt. ratio to prepare the solvent mixture for the ink. Polyvinyl Acetate (PVAc) was added to the solvent mixture in 1:4 wt. ratio and kept at $70^{\circ} \mathrm{C}$ for 30 minutes. A homogenous solution of polymer was prepared. Further, Silver particles were added to this polymer solution in 3:2 wt. ratio. This final mixture was kept under magnetic steering for $3 \mathrm{hrs}$ at $200 \mathrm{rpm}$ and $45^{\circ} \mathrm{C}$ to prepare the Conductive Ink.

The touch sensor was printed using this conductive ink with the screen printing technique (Fig. 1) [4]. A 40 mesh screen was used for the screen preparation. 120-micron thick Polyethylene Naphthalate (PET) film was used as the flexible substrate.

\begin{tabular}{|c|c|}
\hline Parameters & Values \\
\hline Color & Silver \\
Viscosity & $1,038 \mathrm{CPS} @ 25^{\circ} \mathrm{C}\left(50 \mathrm{~s}^{-1}\right.$ Shear Rate $)$ \\
Solid Content & $60 \%$ \\
Density & $2.12 \mathrm{Kg} / \mathrm{L}$ \\
VOC & $0.705 \mathrm{gm} / \mathrm{ml}$ \\
Electrical & $\leq 0.7$ Ohms $/$ Square@20 microns \\
Resistance & \\
\hline
\end{tabular}

Table 1: Properties of Conductive Ink

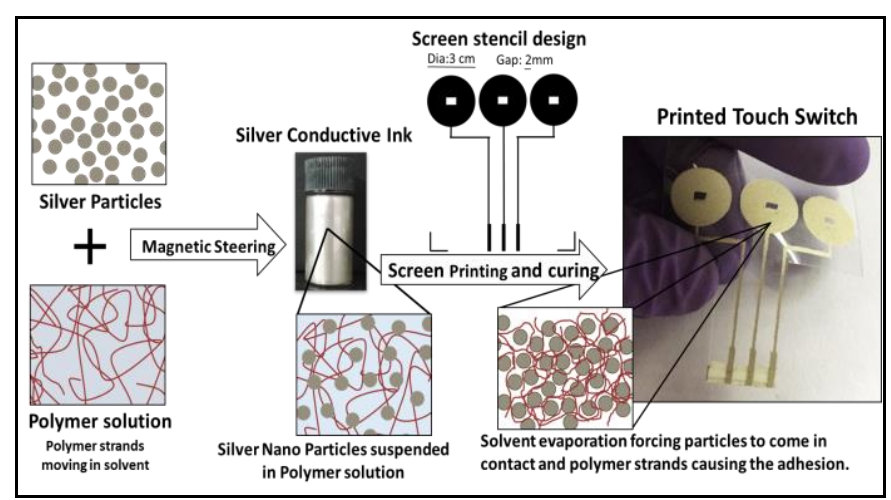

Fig.1. Schematic of Fabrication process 


\section{B. Capacitive touch sensing and acquisition}

In contrast to mechanical switch, the capacitive touch switch requires algorithms and a controller to interpret the signal and determine the on and off sequence. The capacitive sensing algorithm assigns a send pin and a receive pin to the microcontroller [5]. The send pin toggles to a new state and waits for the receive pin to change its state to the same state as the send pin. The algorithm assigns a variable which is incremented in a loop to track the time taken by receive pin to change its state. The delay between the send pin and receive pin is determined by the time constant $(\tau=\mathrm{RC})$, where $\mathrm{R}$ is the resistance applied between the two pins and $\mathrm{C}$ is the receive pin capacitance, plus the capacitance provided when the switch is in proximity with the human body [5]. When the capacitive sensor switch is touched, the finger changes the dielectric constant $(\varepsilon)$ and thus the capacitance $(C=\varepsilon A / d)$, where ' $A$ ' is the cross-sectional area of the plate and ' $\mathrm{d}$ ' is the distance between switch plate and the finger varies which in turn varies the count of time delay tracked by the algorithm, thereby sensing the change in capacitance in term of time delay [5][6][7].

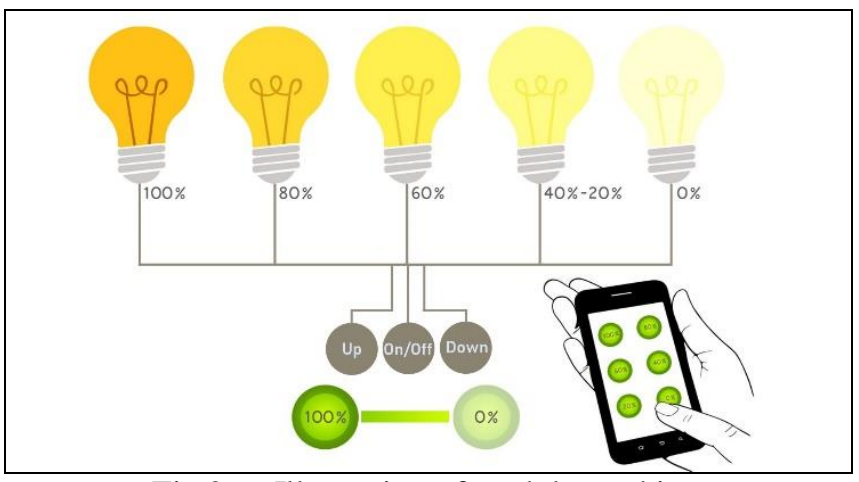

Fig 2. a. Illustration of module working

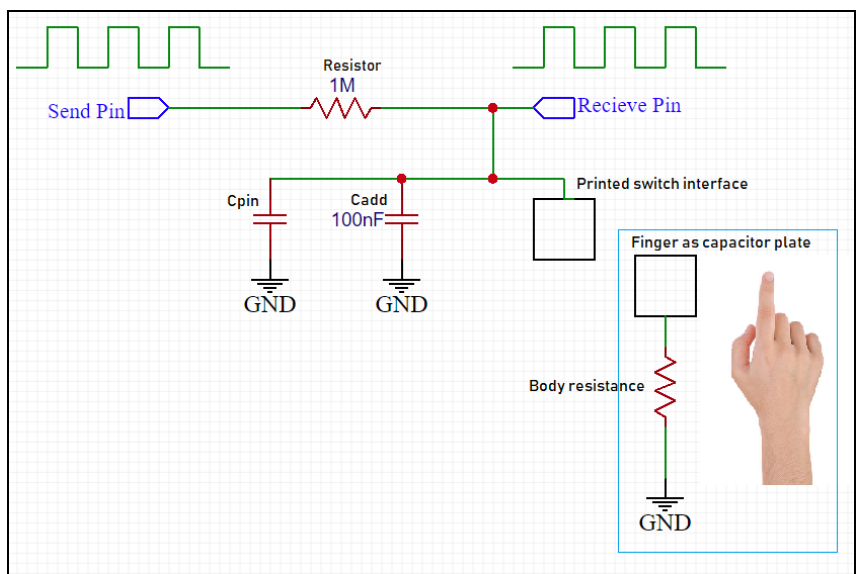

Fig 2.b. Design of capacitive touch sensing method

\section{Wireless data transmission}

In this module, wireless communication is carried out through HC05 Bluetooth module for nearby data transfer and also through ESP8266 Wi-Fi microchip module for IoT based data communication. Several other options include GSM, Ethernet and ZigBee module set, etc. [8].

\section{Module DesigN}

A. Materials, components and specifications

- Silver particles based conductive ink

- Screen printer

- Arduino Pro Mini (5V, $16 \mathrm{MHz})$

- ESP8266 Wi-Fi Microchip Module

- HC05 Bluetooth Module

- Triac optocoupler (MOC3021)

- Transistor optocoupler (4N25)

- TRAIC (BT136)

- MOSFET (IRFZ44N)

- $220 \mathrm{~V}$ AC to $12 \mathrm{~V}$ AC Step down transformer

- IN4007 diodes for rectification and reverse current blocking purpose.

- Passive components (Resistors and capacitors)

- Fuse, Bergs and connectors

- Arduino Genuino software interface

\section{B. Setup}

The proposed module system uses conductive Silver nanoparticle based ink to print flexible switches through screen printing method. The switches are attached to the Arduino pro mini board through flexible printed circuit connector bergs as can be seen in Fig 5. The microcontroller board takes the input from the flexible switch and generates suitable signal which are fed the triac and transistor optocoupler [9]. The optocoupler isolates the microcontroller board from the high voltage mains supply to prevent any damage to sensitive components. The optocoupler provides the replicated gate signal to TRIACs and MOSFETs to control and regulate both $\mathrm{AC}$ and DC loads respectively. The microcontroller board is powered through grid supply itself with the help of $220 \mathrm{~V}$ to $12 \mathrm{~V}$ step down AC transformer followed by rectification and filtering circuits (Fig 3.b) [10].

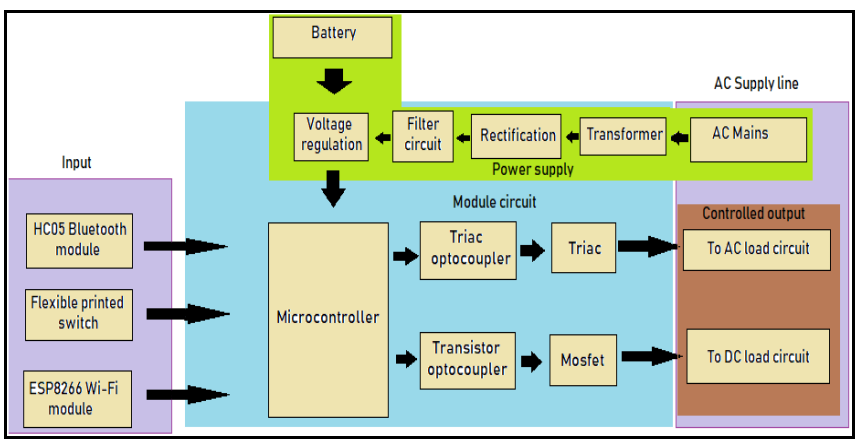

Fig 3.a. Block diagram of designed module

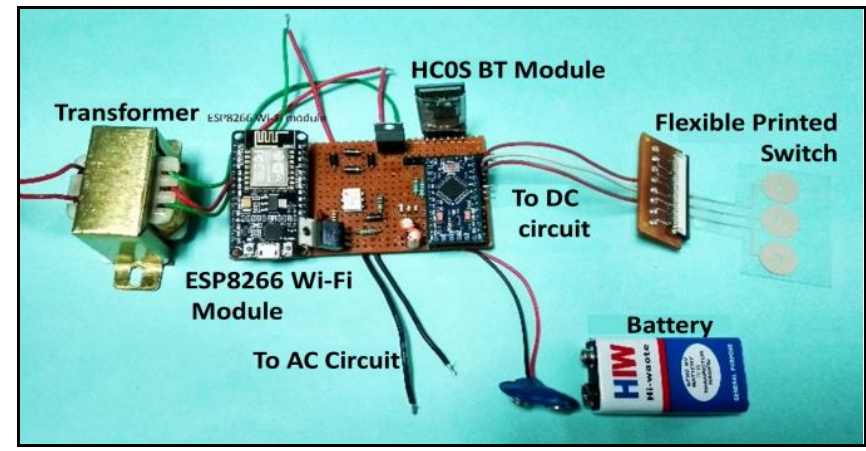

Fig 3.b. Designed through hole dual input power mode module circuit 


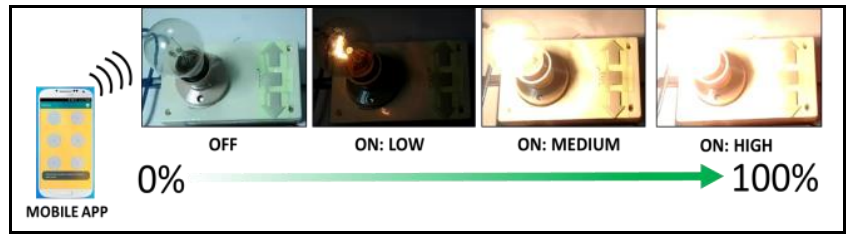

Fig.4. Wireless control of bulb brightness

\section{EXPERIMENTS AND RESULT}

\section{A. Performance analysis of Flexible Printed Switch}

The sensitivity of three flexible switch is tested using Arduino Genuino Serial plotter and the change in capacitance is plotted in terms of incremented variables, with $\mathrm{x}$-axis indicating the timeline and $\mathrm{y}$-axis indicating the time constant value of delay (Fig.5). The output is observed for near proximity by waving the finger 1 to $2 \mathrm{~cm}$ above the switch pad, by tapping, short press and also by long pressing the switch. It is observed that the switch attains a maximum count of 4200 approx. for time delay of signal between send and receive pin measured due to variation in time constant.

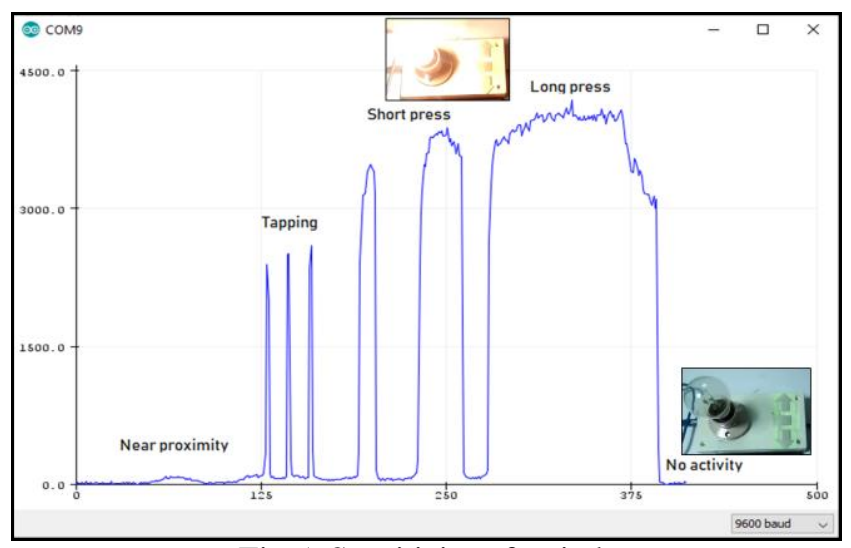

Fig.5. Sensitivity of switch

\section{CONCLUSION}

The paper demonstrates the fabrication of functional foldable flexible switch based on synthesis of high conductivity functional silver ink. The fabricated printed flexible switch was successfully integrated with the microcontroller and wireless module. The designed and integrated system demonstrates IoT readiness for emerging remote access, smart controllers and home automation. Printed, flexible switches demonstrate foldable capacitive sensing, and could be used as an efficient alternative to conventional mechanical and also rigid capacitive sensing switches. The switches were printed using scalable, low cost process providing harmonious prospective integration with the conventional industries for large scale manufacturing. Hence, using printing as the fabrication technique, long arrays of mechanical switches could be replaced just by using single sheet of printed switches which significantly reduces the cost, weight, area requirement and also provides prospective development of touch switches on various flexible and flat substrates such as PET film, glass sheets, wood, ceramic etc. Switches forms the fundamental of electronic system and appliances. Integration of printed switches with wireless module and microcontroller enables seamless evolution of conventional electronic system into smart IoT enabled systems. Printed switches integrated with microcontroller provides a platform to access wireless connectivity and benefits of remote access and automation control of electronic appliances. Hence, as demonstrated and reported here in, these wirelessly controlled smart system may provide prospective use in development of smart controllers and sensors used for home automation, automobile, healthcare, agriculture and developing new age automation and IoT enabled systems.

\section{ACKNOWLEDGMENT}

The authors would like to thank Centre for Energy, IIT Guwahati for providing required laboratory equipment for smooth conduction of experiments, team of $\mathrm{G}$ Labs. Innovation Pvt. Ltd. for helping in development of new design ideas and Department of Design, IIT Guwahati for illustration and development of practical module interface design.

\section{REFERENCES}

[1] K. Baraka, M. Ghobril, S. Malek, R. Kanj and A. Kayssi, "Low Cost Arduino/Android-Based Energy-Efficient Home Automation System with Smart Task Scheduling," 2013 Fifth International Conference on Computational Intelligence, Communication Systems and Networks, Madrid, 2013, pp. 296-301.

[2] J.Chandramohan1, R.Nagarajan2, K.Satheeshkumar3 , N.Ajithkumar4, P.A.Gopinath5, S.Ranjithkumar6, "Intelligent Smart Home Automation and Security System Using Arduino and Wi-fi," International Journal Of Engineering And Computer Science ISSN:2319-7242 Volume 6 Issue 3 March 2017, Page No. 2069420698 Index Copernicus value (2015): 58.10 DOI: 10.18535/ijecs/v6i3.53

[3] S. Kumar and S. R. Lee, "Android based smart home system with control via Bluetooth and internet connectivity," The 18th IEEE International Symposium on Consumer Electronics (ISCE 2014), JeJu Island, 2014, pp. 1-2. doi: 10.1109/ISCE.2014.6884302

[4] Qin, Hantang \& Cai, Yi \& Dong, Jingyan \& Lee, Yuan-Shin. (2016). Direct Printing of Capacitive Touch Sensors on Flexible Substrates by Additive E-Jet Printing With Silver Nanoinks. Journal of Manufacturing Science and Engineering. 139. 10.1115/1.4034663.

[5] Colin H., Jordan H., Ajay K., "Techniques in Swept Frequency Capacitive Sensing: An Open Source Approach" Proceedings of the International Conference on New Interfaces for Musical Expression, NIME'14, June 30 - July 03, 2014, Goldsmiths, University of London, UK.

[6] Zuk, S., Pietrikova, A. and Vehec, I. (2018), "Capacitive touch sensor", Microelectronics International, Vol. 35 No. 3, pp. 153157. https://doi.org/10.1108/MI-12-2017-0071

[7] J. T. Kung, H. -. Lee and R. T. Howe, "A digital readout technique for capacitive sensor applications," in IEEE Journal of Solid-State Circuits, vol. 23, no. 4, pp. 972-977, Aug. 1988. doi: $10.1109 / 4.348$

[8] K. J. Singh and D. S. Kapoor, "Create Your Own Internet of Things: A survey of IoT platforms.," in IEEE Consumer Electronics Magazine, vol. 6, no. 2, pp. 57-68, April 2017. doi: 10.1109/MCE.2016.2640718

[9] Rand, Dustin, et al, Issues, Models and Solutions for Triac Modulated Phase Dimming of LED Lamps, Power Electronics Specialists Conference, 2007. PESC 2007. IEEE, Jun. 17-21, 2007, pp. 13981404, Boston, MA, USA

[10] Azoteq, IQS17 Family, IQ Switch-ProxSense Series, Touch Sensor, Load Control and User Interface, IQS17 Datasheet V2.00.doc, Jan. 2007, pp. 1-51, Azoteq (Pty) Ltd., Paarl, Western Cape, Republic of South Africa 